\title{
La temporalidad del canon y su construcción en la historia del arte a través del museo de copias en el siglo XIX
}

\section{The Temporality of the canon and Its Construction in the History of Art Through Museum of Copies During the $19^{\text {th }}$ Century}

\author{
Milena Gallipoli \\ Centro de Investigaciones en Arte y Patrimonio (CIAP), \\ CONICET-UNSAM (Argentina) \\ mgallipoli@unsam.edu.ar
}

\begin{abstract}
Resumen
En el siglo XIX se asistió a una importante institucionalización de las artes gracias a la fundación de los museos de copias de obras maestras en yeso. Este trabajo examina de qué modo el museo de reproducciones artísticas ha visibilizado, construido y activado el relato canónico del arte. En este artículo defendemos la hipótesis de que fue este un lugar idóneo para el nacimiento de una historia que se basó en la combinación de la idea de historia magistra vitae y del moderno concepto de progreso. A través de la historia de los conceptos de canon y clásico se examina la aplicación de tales categorías a la esfera de la formación artística.
\end{abstract}

\section{Palabras claves}

Historia del arte, museo, calcos escultóricos, canon, clásico, pasado histórico-artístico.

\begin{abstract}
The nineteenth century was witness of a crucial institutionalization in the arts thanks to the foundation of museums of copies of sculptural masterpieces in plaster cast. This article examines in what manner the museum of artistic reproductions visualized, constructed and implemented the canonical account of art. We defend the hypothesis that this institution was the most suitable place to harbour a vision of history which turned out to be a mix between the idea of historia magistra vitae and the modern concept of progress. Through the history of concepts such as canon and classical we shall study the application of these categories to the realm of artistic education.
\end{abstract}

\section{Keywords}

History of art, museum, plaster casts, canon, classical, historical-artistic past. 
Entre los siglos XVIII y principios del XX, en paralelo a la aparición del museo artístico, hubo un proceso de creación de colecciones de copias en museos, academias y universidades. Especialmente hacia fines del siglo XIX este tipo de instituciones alrededor del mundo fueron sumamente prolíficas gracias a la disponibilidad y el bajo costo de las reproducciones, particularmente aquellas de esculturas famosas que se realizaban en yeso, llamadas calcos escultóricos. ${ }^{1}$

El objetivo del presente trabajo es analizar las dinámicas de construcción de una historia del arte tomando como punto de partida los casos de los museos de copias escultóricas de obras de arte, generalmente llamados museo de escultura comparada, de calcos o de reproducciones artísticas, a través del siglo XIX. Dada la reproductibilidad, el museo de calcos reúne en un mismo espacio una serie de obras canónicas que se repiten; ni la originalidad ni la unicidad son los criterios que guían la conformación de estas colecciones. Asimismo, la utilidad y el uso de estas copias está intrínsecamente ligado a la formación y la educación, tanto del estudiante de artes como del público en general, ya que los calcos escultóricos eran concebidos como modelos objetuales funcionales a la "elevación del gusto".

Por ejemplo, en 1885 el pintor y gestor cultural argentino Eduardo Schiaffino describía el Musée des études de la École des Beaux-Arts de París en los siguientes términos:

Si enumero aunque superficialmente las reproducciones fieles de las obras de arte de todos los siglos y de todas las escuelas, que permiten a los habitantes de esta capital hacerse una idea del arte en general y estudiarlo sin salir de París con sólo frecuentar la Escuela de que hablo, es a fin de mostraros la verdad de mi aseveración al reconocerle a l'École des BeauxArts la primacía entre las Academias del mundo. ${ }^{2}$

El espacio era un amplio patio interno vidriado que contenía copias de las más importantes esculturas de la Antigüedad -desde unas monumentales columnas del Templo de Castor y Pólux hasta la Venus de Milo- y exhibía calcos de otros periodos artísticos - desde la Edad Media hasta la escultura moderna. En ese entonces, la ciudad europea se había convertido en uno de los más prominentes centros de producción y formación artística, siendo la École la institución de más prestigio para la educación de las artes. ${ }^{3}$ Allí, se combinaba la modalidad de uso de academia con museo, dado que uno

\footnotetext{
${ }^{1}$ Recientemente se ha desarrollado un vasto corpus bibliográfico referente al coleccionismo institucional de calcos escultóricos alrededor del mundo. Algunas referencias básicas son: Rune Frederiksen y Eckart Marchand (eds.), Plaster Casts: Making, Collecting, and Displaying from Classical Antiquity to the Present (Berlin: Walter de Gruyter, 2010); Charlotte Schreiter, Gipsabgüsse und antike Skulpturen. Präsentation und Kontext (Berlin: Reimer, 2012); José Beltrán Fortes, Luis Méndez Rodríguez, y José María Luzón Nogué, Yesos: Gipsoteca de la Universidad de Sevilla (Sevilla: Centro de Iniciativas Culturales de la Universidad de Sevilla, 2015) y el volumen 28 del año 2016 la revista In Situ. Revue de patrimoines. Le moulage. Pratiques historiques et regards contemporains. Específicamente en América hubo un asiduo consumo de calcos escultóricos a lo largo del siglo XIX y principios del XX, se formaron colecciones en Argentina, Uruguay, Chile, Brasil, Colombia, México, Estados Unidos, Canadá, por sólo nombrar algunos países. Este aspecto en particular fue el objeto de estudio de mi tesis de maestría titulada Las rutas del yeso. Circulación y consumos globales de calcos escultóricos hacia fines del siglo XIX (Tesis de maestría, IDAES, UNSAM, 2017).

${ }^{2}$ Eduardo Schiaffino, "El arte en Paris I", El diario (Buenos Aires), 18 de marzo de 1885 en Laura Malosetti Costa (selec.), Cuadros de viaje. Artistas argentinos en Europa y Estados Unidos (1880-1910) (Buenos Aires: Fondo de Cultura Económica, 2008), 159.

${ }^{3}$ Hay un amplio corpus bibliográfico que ha abordado la historia y el análisis de esta institución, sólo por nombrar algunos de los estudios más completos y específicos véase Alain Bonnet, L'enseignement des arts
} 
de los aspectos más destacados del espacio era esta gran exhibición de reproducciones junto con trabajos de los estudiantes que eran utilizados por los inscriptos y aspirantes para copiar y estudiar estética e historia del arte. En un mismo lugar, aquellas obras de arte consideradas como obras maestras, se hacían disponibles para que el espectador pudiese aprehenderlas a través de la práctica y la teoría. Estas lecciones del pasado eran los modelos que guiarían a los grandes artistas del presente y del futuro.

THE PARIS SCHOOL OF FINE ARTS.

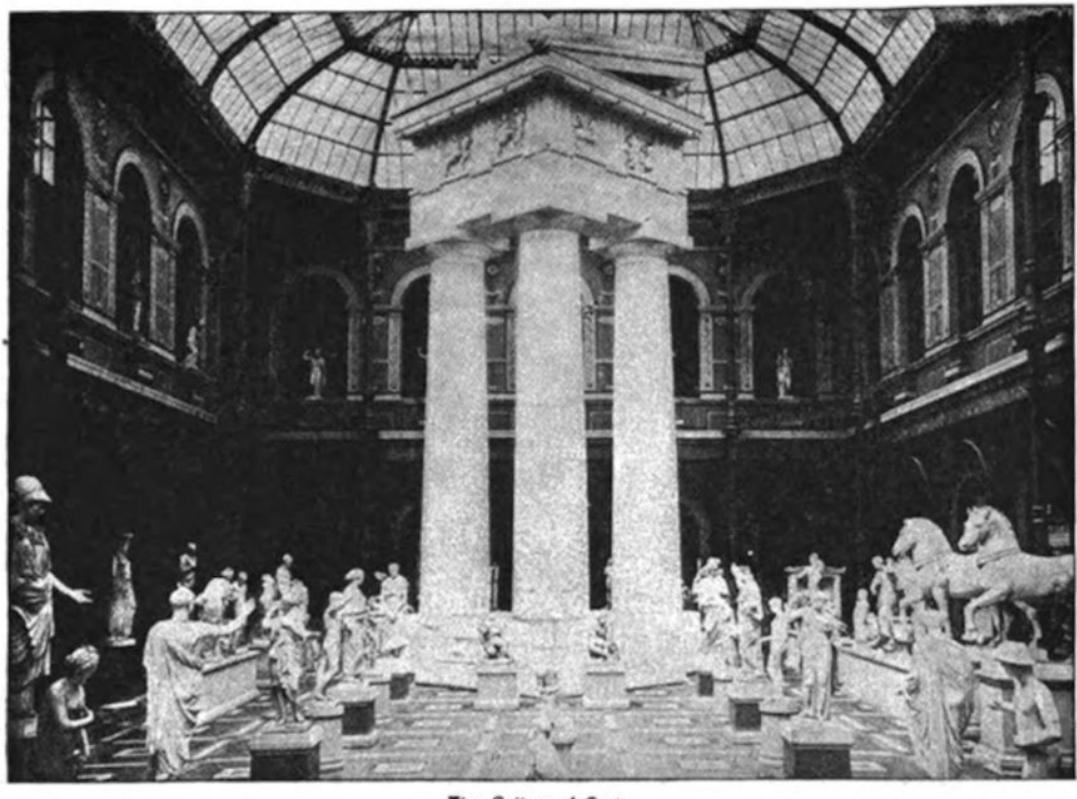

Fotografía publicada en Avery, Henry O.,

“The Paris School of Fine Arts”, Scribner's Magazine, 4 (II) (1887), 399.

En este sentido, surge un concepto clave para la historia del arte que vertebrará la presente argumentación: la categoría de canon. Particularmente, se examinará cómo el canon artístico construye y consolida una historia del arte y qué relación establece ésta con la temporalidad. De esta forma, se analiza la construcción que el museo de calcos hace de la historia del arte como un relato canónico de obras que demuestran en su reunión el progreso en función de su enseñanza para fomentar la práctica artística del momento.

El historiador alemán Reinhart Koselleck ha señalado que la forma en que los hombres viven y cuentan la historia está en función de cómo se comprende el tiempo. ${ }^{4}$ Es por esa razón que este trabajo también tiene como objetivo indagar la utilidad de la historia conceptual y las propuestas realizadas por Koselleck y François Hartog respecto a la temporalidad para examinar qué concepción del tiempo y del devenir histórico

au XIXe siècle. La réforme de l'École des Beaux-Arts de 1863 et la fin du modèle académique (Rennes: Presses Universitaires de Rennes, 2006); Emmanuel Schwartz, La chapelle de l'École des Beaux-Arts de Paris (Paris: École Nationale Supérieure des Beaux-Arts, 2002) y Monique Segré, L'art comme institution. L'École des Beaux-Arts, 19e et 20e siècles (Cachan: Editions de l'École Normale Supérieure de Cachan, 1993).

${ }^{4}$ Reinhart Koselleck, Historia y hermenéutica (Barcelona: Paidós, 1997), 28. 
pretende mostrar el museo de calcos. ${ }^{5}$ Por esa razón, la argumentación se mantendrá en un plano teórico y conceptual, aunque con el objeto de estudio como horizonte de análisis.

La cuestión del tiempo atraviesa intrínsecamente la conformación de estas colecciones dado que es un explícito armado del pasado, pero que a su vez revela una concepción implícita de un determinado relato sobre aquél, que se halla generalmente naturalizado e incorporado. Por esa razón, algunas de las reflexiones metodológicas sobre el análisis de fuentes de Koselleck serán de suma utilidad al momento de examinar la presente pregunta de investigación. En este sentido, el autor plantea:

La investigación histórica que se involucra con preguntas factuales no tiene que explícitamente plantear la cuestión del tiempo histórico. En adición, las fuentes "de" determinado tiempo raramente proveen alguna información directa "sobre" ese tiempo. Por lo tanto, debemos clarificar nuestras preguntas teóricamente en función de hacerlas operacionales para con la investigación. ${ }^{6}$

Basándose en este planteamiento, el trabajo se concentrará en la relación que el concepto de canon y la categoría de lo clásico establecen con la temporalidad para ver qué tipo de historia construye el museo de copias. Por último, pretendemos examinar la temporalidad a la luz del concepto de régimen de lo histórico de Hartog para dar cuenta de la conjugación entre pasado, presente y futuro en relación con nuestro objeto de estudio.

De esta forma, se reflexionará sobre el devenir histórico de las copias escultóricas de obras arte en sus contextos atendiendo a sus relaciones con el tiempo. También, se explora la vinculación entre ciertas prácticas de la esfera artística y algunos conceptos nodales de la historia del arte.

En este sentido, la historia conceptual planteada por Koselleck afirma que los conceptos tienen una estructura temporal dados por una dimensión semántica y una pragmática. Sintéticamente, mientras que la primera refiere a los significados consolidados de un concepto, la segunda se relaciona con sus usos particulares en un determinado recorte de espacio y tiempo. ${ }^{7}$ Por ende, indagar en la dimensión pragmática

\footnotetext{
${ }^{5}$ Cabe destacar que tanto Koselleck como Hartog han tenido un activo interés por las artes visuales y la historia del arte. Por un lado, Koselleck ha dedicado algunos ensayos a cuestiones artísticas a partir de casos de estudios como los monumentos de guerra y el dibujante francés Honoré Daumier (ensayos contenidos en Reinhart Koselleck, The practice of conceptual history. Timing history, spacing concepts (Stanford: Stanford University Press, 2002), 265-326). Asimismo, la imagen ha formado parte integral de su forma de argumentar, ejemplificado a través de su emblemático análisis del cuadro de la batalla de Issus de Albrecht Altdorfer (1529) en Futuro, pasado (Reinhart Koselleck, Futuro, pasado (Barcelona: Paidós, 1993)). Por otro lado, Hartog se dedicó a desarrollar el concepto de patrimonialización en función de su análisis de la relación entre historia y memoria (François Hartog, "El presente y el historiador," Anuario TAREA, 1 (2012): 37 (27-41)) y ha tomado episodios de la historia del museo del Louvre como ejemplos argumentativos en su libro Regímenes de historicidad (François Hartog, Regímenes de historicidad. Presentismo y experiencias del tiempo (México: Universidad Iberoamericana, 2007)).

${ }^{6}$ Reinhart Koselleck, The practice of conceptual history, 111.

${ }^{7}$ Para una explicación más profunda de estos conceptos véase: José Javier Blanco Rivero, "La historia de los conceptos de Reinhart Koselleck: conceptos fundamentales, Sattelzeit, temporalidad e histórica," Politeia, 35, 49 (2012), 12. Igualmente el propio Koselleck advierte que: "el significado y el uso de una palabra nunca establece una relación de correspondencia exacta con lo que llamamos la realidad. Ambos, conceptos y realidades, tienen sus propias historias que, aunque relacionadas entre sí, se transforman de diversas maneras". Reinhart Koselleck, "Historia de los conceptos y conceptos de historia," Ayer, 53 (2004), 36.
} 
del concepto de canon en función del caso del museo de copias, permitirá a su vez reflexionar sobre su dimensión semántica.

\section{El canon, lo clásico, y su relación con el tiempo}

Originalmente, el concepto de canon apareció en la Antigüedad como título del tratado que inauguró un sistema de proporciones del escultor Policleto de Argos. El canon pasó a significar, en el ámbito del arte, aquellas formas y proporciones que son universalmente aceptadas por los artistas como obligatorias y esenciales para acceder a las formas bellas. ${ }^{8}$

El canon posee la peculiaridad de que, si bien varía históricamente, en su momento de instauración necesita imponerse como universal y eternamente válido, de modo que fijeza y objetividad son caracteres esenciales para que adquiera una entidad ontológica de verdad. En la institución del museo, el canon se encarna a través de la obra maestra: una pieza ejemplar eterna, que es y será maestra, y por ende debe ser conocida, estudiada y comprendida.

Esta noción que remite a las obras de arte individuales, se extendió a la categoría de estilo, por ejemplo, una serie de rasgos en común que poseen las piezas que hacen que se puedan reunir en escuelas artísticas, definidas en general por el tiempo y el espacio nacional en el que se produjeron. Esta idea de escuela, que nuevamente remite a la de formación, educación y ejemplaridad, fue el criterio de exhibición que determinó la disposición de las obras en los museos, desde el siglo XVIII hasta la actualidad inclusive. ${ }^{9}$ Así, el museo fue el depósito del gran arte de las obras canónicas junto con el resto del conjunto de la escuela artística, inaugurando un criterio de conocimiento del devenir del arte en clave comparativa y ejemplar. Como dice el historiador del arte francés Éric Michaud: "El público (ciudadanos de la nación) era invitado a comparar las supuestas cualidades propias de cada una de las escuelas nacionales, es decir, a elaborar en, y gracias al espacio del museo, una cartografía de los «caracteres nacionales»". ${ }^{10}$

Si el museo se caracterizó por presentar y escenificar la historia del arte a partir de las escuelas artísticas y sus piezas destacadas, el museo de escultura comparada replicaba esta misma operación a través de la repetición y condensación de estos ejemplares en un muestrario que tomaba las obras maestras de cada colección alrededor del mundo. La reproductibilidad permitía el encuentro de obras canónicas de varios espacios y tiempos en un mismo lugar. ${ }^{11}$

\footnotetext{
${ }^{8}$ El filósofo e historiador de la estética polaco Wladislaw Tatarkiewicz reseña y examina la dimensión semántica y su desarrollo histórico tanto del concepto de canon como del de clásico. Véase: Wladislaw Tatarkiewicz, Historia de la estética I: La estética antigua (Madrid: Akal, 1987) y Historia de seis ideas. Arte, belleza, forma, creatividad, mímesis, experiencia estética (Madrid: Tecnos, 2001).

${ }^{9}$ Éric Michaud examina la noción de escuela artística y su relación con el gusto y la nación. En su libro Las invasiones bárbaras... realiza un análisis en donde conjuga la historia conceptual con algunas prácticas institucionales como la del museo. Afirma que: "No había sino a partir de fines del siglo XVIII que se había impuesto lentamente, en el seno de las galerías de pintura y luego en los museos de Europa, un modo de exposición de las obras por escuelas nacionales" (Éric Michaud, Las invasiones bárbaras. Una genealogía de la historia del arte, trad. De Antonio Oviedo (Buenos Aires: Adriana Hidalgo, 2017), 50).

${ }^{10}$ Éric Michaud, Las invasiones bárbaras, 53.

${ }^{11}$ Cabe aclarar que estas operaciones llevadas a cabo por el museo y por el museo de copias no necesariamente tienen que ver con una cantidad determinada de piezas. En general, a lo largo del siglo XIX y hasta las instauración de la denominada museografía de la "estética del cubo blanco" el criterio fue una suerte de "cuanto más, mejor". No obstante, esta proliferación cuantitativa no quiebra la efectividad del
} 
El canon debe difundirse, y esto sucede a través de la operación de la copia. Así, la constante replicación de las obras de arte -que se yerguen como únicas y estimadas- es lo que permite que el canon sea dinámico y constantemente difundido y reforzado. De esta forma, canon y copia, dos términos que a primera vista podían parecer contradictorios, se hallan en mutua dependencia. El calco escultórico es un objeto perteneciente a la esfera de lo no canónico, pero que, junto a otros múltiples dispositivos, otorga solidez a la posición del canon artístico. De esta forma, podemos notar la compleja relación que se establece entre el concepto de canon y cómo fue puesto en práctica por parte de la institucionalidad del museo tradicional y el de copias.

Si bien, en principio, el concepto de canon no refiere a un tiempo ni espacio en particular, sino antes bien a una suerte de relato histórico completo compuesto por una sucesión de estilos y escuelas, la asociación entre el canon y lo clásico, ha surgido como una yuxtaposición casi natural. Desde el principio, la noción de clásico también estuvo asociada a un valor, a una caracterización de excelencia, pero, además, se vinculó al periodo de la Antigüedad.

Koselleck en Begriffsgeschichten (2006) analiza brevemente la relación que el tiempo establece con el tipo de fuentes denominadas por él como textos clásicos. Según el autor, un clásico es una fuente del pasado que adquiere un valor de eternidad, en este sentido, podemos agregar, encaja con la lógica de funcionamiento de lo canónico. "Los mensajes que contienen los textos clásicos apuntan a la afirmación de verdades permanentemente válidas a las que siempre se puede recurrir $\mathrm{y}$, por lo tanto, a una repetibilidad potencialmente infinita". ${ }^{12}$ Más aún, esta relación entre el tiempo y el clásico posee una estructura conflictiva porque "un texto clásico cumple la paradoja de ser único y, por el contrario, debe leerse para una repetición duradera. ${ }^{13}$

Ahora bien, hemos reseñado brevemente una serie de acepciones de la categoría de lo clásico que se han concentrado en los textos como principal objeto de estudio, ${ }^{14}$ de modo que cabe interrogar qué sucede cuando la pregunta se desplaza hacia la esfera de las artes visuales. En este sentido, el arqueólogo e historiador del arte italiano Salvatore Settis en su libro El futuro de lo clásico problematiza que la pretendida y construida eternidad del concepto ha radicado en la asimilación de la historia griega a la historia universal. Allí afirma que:

Clásico es de por sí un concepto estático, pues designa un periodo histórico que por definición está concluido; el cual, sin embargo, no tiene sentido y no se vuelve operativo sin un mecanismo dinámico de nostalgia y de iteración, sin una cierta pulsión bien hacia el retorno a lo "clásico", bien a su superación. En otros términos, "clásico" y "clasicismo" forman una pareja de conceptos que se explican y legitiman mutuamente. ${ }^{15}$

concepto de canon dado que otra de las acepciones del concepto es la relación proporcional de la parte con el todo, de modo que, siempre y cuando hubiera una unidad de clasificación, que en este caso es la de escuela artística, todas las piezas encajarían con el relato canónico.

${ }^{12}$ Reinhart Koselleck, Begriffsgeschichten (Frankfurt am Main: Suhrkamp, 2006), 98.

13 Íbid., 98.

${ }^{14}$ Sobre la tradición clásica en literatura véase Gilbert Highet, The Classical tradition. Greek and Roman influence in western literature (Oxford: Oxford University Press, 1985) y Craig W. Kallendorf, A companion to the classical tradition (Oxford: Blackwell publishing, 2007).

${ }^{15}$ Salvatore Settis, El futuro de lo clásico (Madrid: Abada, 2006), 26. 
En el campo artístico, la reunión de obras maestras originales en el museo se complementó con la cristalización y difusión de un sistema académico clasicista de formación artística que estableció una forma dominante de mirar y de copiar. Allí, la presencia de copias en yeso determinaba y fijaba cuáles eran los modelos maestros, ${ }^{16}$ y la obra clásica era una fuente de conocimiento con estatuto de verdad que estaba por encima de todas las escuelas artísticas e, inclusive, conformaba sus bases y fundamentos. Abrevar en ese lugar común, lejos de ser un plagio, era una forma de ser original. Así, se valora la originalidad de la semejanza como mecanismo dinámico de activación y, en relación con lo antedicho, cabe discutir el carácter nostálgico que le atribuye Settis al clasicismo, problemática que por el momento sólo dejaremos planteada para retomarla más adelante en la argumentación.

Esta intersección entre indicadores históricos y el concepto es una de las problemáticas que la historia conceptual ha abordado al reclamar la necesidad de una vinculación activa entre la historia social y la historia conceptual. La comprensión del tiempo está mediada por su construcción, y ese constructo se erige de forma histórica: "El tiempo histórico [...] está atado a unidades políticas de acción, [...] y a sus instituciones y organizaciones. Todos tienen un cierto modo inherente de desempeño, de las que cada una posee su propio ritmo temporal". ${ }^{17}$

En este sentido, el museo tradicional y el de copias en su conjunción estratégica con la escuela artística y/o academia establece una temporalidad propia a los tiempos de la formación. Eran las instituciones que educaban a los artistas y al público, que proveían los fundamentos del arte, lo que implica una determinada relación con el tiempo y el pasado muy diferente a la lógica de la producción artística y al mercado, en donde la categoría de innovación y novedad tiene otra importancia. De esta forma, incluso nos encontramos ante diferentes ritmos temporales dentro del campo artístico. Ahora bien, cabe preguntarte, ¿cómo se ponía en juego la temporalidad en la formación artística?

\section{Pasado-Futuro. Dinámicas de perpetuación y difusión del canon artístico}

En principio, se puede establecer que en las artes, el origen y el principio se encuentra en la antigüedad grecorromana. Retomando la problemática del concepto de canon y clásico, François Hartog afirma la pareja de los Antiguos y los Modernos ha estructurado en profundidad, y en larga duración, la relación entre el tiempo y la historia de la cultura occidental. ${ }^{18}$ Asimismo, Settis refuerza y especifica este planteo en la prioridad cultural de Grecia que ha funcionado como una "cuna originaria" de valores y de ideas clásicas $-\mathrm{y}$ occidentales. ${ }^{19}$

\footnotetext{
${ }^{16}$ Entendemos el sistema clasicista a partir de la definición y análisis que el historiador del arte Richard Shiff hace al respecto. Define al clasicismo como un sistema que desarrolla ciertas normas o estándares para la representación de la realidad que se perpetúan a través de formas de reproducción, como la copia. Así, una representación dada es estimada hasta el grado de que es considerada verdadera o adecuada a su modelo, estableciendo así un 'lugar común' [commonplace] de la verdad clasicista. Véase Richard Shiff, "The original, the imitation, the copy and the spontaneous classic: Theory and painting in Nineteenthcentury France," Yale French Studies, 66 (1984), 27-54 y "Phototropism (Figuring the proper)", Studies in the History of Art, 20 (1989), 161-179.

${ }^{17}$ Reinhart Koselleck, The practice of conceptual history, 110.

${ }^{18}$ François Hartog, Regímenes de historicidad, 91. Cabe agregar que, según el autor, otro de los conceptos fundamentales es el del salvaje.

${ }^{19}$ Salvatore Settis, El futuro de lo clásico, 20.
} 
Es claro que estos conceptos han atravesado de forma nodal a gran parte de la historia del arte ${ }^{20}$ y se han cristalizado a partir de los escritos de Johann Joachim Winckelmann, que se podrían en parte condesar a través de su famosa frase: "El único camino que nos queda a nosotros para llegar a ser grandes, incluso inimitables si es posible, es el de la imitación de los antiguos". ${ }^{21}$ No es casual que Michaud afirme: "Es con Winckelmann que «comienza la historia del estilo»". ${ }^{22} \mathrm{Si}$ bien la pregunta de este trabajo no hace pertinente concentrarnos en el rol que Winckelmann cumplió en relación con la historia del arte y el clasicismo en general, ${ }^{23}$ cabe mencionar brevemente que su libro Reflexiones sobre la imitación del arte griego en la pintura y la escultura establecía un complejo vínculo entre pasado-Antigüedad y presente-moderno dado por la crítica negativa del arte del momento, que sólo podría ser redirigido de forma positiva -con una mirada retrospectiva de tipo nostálgica, retomando las palabras de Settis- hacia la Antigüedad como modelo prescriptivo a imitar. La relativa sincronía entre los escritos del connoisseur alemán y el nacimiento y la proliferación del sistema académico de enseñanza artística será una conjunción extra en pos de la consolidación de este modelo canónico formativo.

En el recorte temporal planteado del siglo XIX, la práctica de copiar era una forma de desarrollar el poder de invención del estudiante mediante la aprehensión de los principios compositivos y recursos plásticos de los grandes maestros, encarnados en determinadas obras maestras -en su mayoría, aquellas pertenecientes a la Antigüedad clásica. Como resume el historiador del arte norteamericano Richard Shiff en relación con el sistema clásico:

\begin{abstract}
Se abogó por una copia hecha a mano como un dispositivo para revelar tanto el genio del copiado como el del copista. Concibieron que la fuerza espiritual del maestro clásico se transfería al lienzo a través de la mano del maestro moderno en un acto de asimilación consumada. Este fue el tipo de 'manejo' que aseguraría la vitalidad de la tradición clásica. Preservaba el pasado sin negar el presente. ${ }^{24}$
\end{abstract}

A través del dibujo, los estudiantes en formación debían imitar otras obras canónicas, la pregunta recae sobre qué modelos eran los adecuados a mirar. Hemos establecido anteriormente la relación entre escuela artística y obra maestra. Por un lado, cada escuela artística representaba un estilo, se delimitaba como tal por la reunión de una serie de rasgos en común tanto históricos -coincidencia espacio y tiempo- como formales. Asimismo, se suponía -si el proceso era exitoso- que cada artista en formación pasaría a integrarse y representar una determinada escuela artística, que en el siglo XIX,

\footnotetext{
${ }^{20}$ Sólo por nombrar algunos títulos entre el amplio corpus bibliográfico que ha lidiado con esta temática véase Erwin Panofsky, Renacimiento y renacimientos en el arte occidental (Madrid: Alianza, 1997); Francis Haskell y Nicholas Penny, Taste and the Antique: The Lure of Classical Sculpture. 1500-1900 (New Haven: Yale University Press, 1981) y Elaine K. Gazda (ed.), The Ancient Art of Emulation. Studies in Artistic Originality and the Tradition from the Present to Classical Antiquity (Michigan: The University of Michigan Press, 2000).

${ }^{21}$ Johann Joachim Winckelmann, Reflexiones sobre la imitación del arte griego en la pintura y la escultura (Barcelona: Nexos, 1987 [1755]), 18.

${ }^{22}$ Éric Michaud, Las invasiones bárbaras, 38.

${ }^{23}$ Sobre esta temática véase Éric Michaud, Las invasiones bárbaras; Alex J. Potts, "Greek Sculpture and Roman Copies I: Anton Raphael Mengs and the Eighteenth Century," Journal of the Warburg and Courtland Institutes, 43 (1980), 150-173; Francis Haskell y Nicholas Penny, Taste and the Antique y Hans Belting, The invisible masterpiece (London: Reaktion books, 2001).

${ }^{24}$ Ricard Shiff, "Phototropism (Figuring the proper)", 67.
} 
se vinculó fuertemente con el criterio nacional y racial. De esta forma, ese individuo debía plasmar en sus obras esa serie de rasgos característicos propios de su nación y raza. ${ }^{25}$

Ahora bien, por otro lado, en la instancia formativa, ni la originalidad ni la búsqueda del propio estilo se presentaban como los criterios de valor sino antes bien la correcta adecuación al canon a través de la copia literal de las academias por un lado, y la presentación de obras relativamente inventivas para los concursos, por el otro. Por esa razón, en esa etapa, el museo de reproducciones artísticas actúa con un doble filtro selectivo de modelos a imitar. En primer lugar, hay una selección inicial de un relato de la historia del arte que tendía a privilegiar una presentación lo más completa del devenir de los diversos estilos a través del tiempo. Esto era lo que Schiaffino declaraba sobre la colección de la École en París y que también por ejemplo afirmaba de forma similar un artículo periodístico de 1887 publicado en Estados Unidos: "Estos calcos, en interminables cuartos, halls y corredores, todos de gran tipo, aparecen como maestros mudos, que parecen decirle al estudiante: «En nosotros están contenidos los secretos de la belleza y la proporción, y la más alta expresión del verdadero arte...»". ${ }^{26}$

Pero luego, hay un segundo momento: entre ese muestrario relativamente inclusivo se extraen aquellas piezas que deben ser copiadas por los estudiantes, y allí es cuando el canon clásico hace su aparición con más fuerza. En la operación de la copia de copias se da la mayor selectividad del pasado y es cuando el canon actúa de forma más restrictiva. Más aún, allí la efectividad de lo clásico depende de su confusión y unión con lo universal, se accede a la belleza universal como lección del pasado en función de formar, fomentar y propiciar que el artista pueda producir "la belleza del futuro" y llevar progreso a su escuela artística respectiva. Así, particular-general, antiguo-moderno y pasado-futuro se amalgaman en un sistema de funcionamiento de relativa coherencia que es reforzado por la dinámica dogmática del canon. De esta forma, parafraseando a Settis, podemos notar que la operación llevada a cabo, no responde a una lógica dinámica nostálgica de rescate de un pasado irremediablemente estático por acabo, sino antes bien a una operación dinámica de apropiación e incorporación de ese pasado, que vuelve a comenzar ante cada rescate. El artículo estadounidense lo decía de forma clara en la continuación del discurso ficcional de los calcos al estudiante:

\begin{abstract}
No nos copien, pero recuerden las leyes que nos controlan; no nos imiten, sino creen, al retornar a la simplicidad y la grandeza, mientras escriben naturalmente, y sin artificio ni pretensión, esa lengua universal llamada arte. Sean pensadores; y, sobre todo, mantengan en Francia esa iniciativa que, en las artes, ha procurado para ella tantas victorias y tal supremacía constante. $^{27}$
\end{abstract}

Esta frase en parte sintetiza lo que hemos estado señalando: si bien se pregona una copia de tipo literal, ésta no implica una renuncia de la subjetividad en pos de una imitación servil sino que antes bien se inserta en una lógica activa de formación artística. Son copias de obras canónicas que actúan de maestras para que el estudiante se pueda convertir en artista y contribuir al arte de su escuela-nación que, no es casual que en el caso de la fuente periodística, se ejemplifica por la hegemonía cultural de Francia. De esta forma, podemos establecer un pequeño desplazamiento en el sentido de clasicismo

\footnotetext{
${ }^{25}$ Cabe agregar lo que afirma Michaud al respecto: "El gusto se forma y es formador. Si puede ser formado a la escala colectiva de una nación igual que la del individuo singular, permanece siempre con un alcance eminentemente social" (Éric Michaud, Las invasiones bárbaras, 29).

${ }^{26}$ Henry O. Avery, “The Paris School of Fine Arts”, Scribner's Magazine, 4 (II) (1887), 397-398.

27 Íbid., 398-399.
} 
para dejar de comprenderlo como una serie de oleadas estilísticas dedicadas al rescate del pasado antiguo clásico - como lo ha planteado Settis- y concebirlo como la permanencia de una serie de prácticas que han subyacido al sistema formativo artístico por un periodo de larga duración. ${ }^{28}$ Como un concepto, entendido desde la historia conceptual, se puede decir que se debe cotejar esta larga duración y permanencia con cambios específicos a lo largo del tiempo y adaptaciones y modificaciones múltiples a través del espacio.

\section{¿Un régimen de historicidad artístico? El arte entre la historia magistra vitae y el progreso}

Hasta el momento, hemos estado examinando las formas en que el pasado, el presente y el futuro se conjugan en la formación artística en el marco de la escuela o academia y la instrumentalización que ésta hace del museo de calcos para presentar una historia del arte. Como sintetiza Koselleck podríamos agregar que: "en todos los casos se pregunta cómo en cada momento presente las dimensiones temporales del pasado y del futuro se remiten las unas a las otras". ${ }^{29}$

En este sentido, surge la necesidad de detenerse en el concepto de régimen de historicidad planteado por Hartog en su libro Regímenes de historicidad. Presentismo y experiencias del tiempo (2007). Sintéticamente, el historiador define que "un régimen de historicidad sólo es una manera de engranar pasado, presente y futuro o de componer una mixtura de tres categorías", ${ }^{30}$ y que debe ser utilizada como una herramienta heurística. Más allá de las utilidades metodológicas y los planteos conceptuales que suscita la noción de régimen de historicidad, cabe examinar más detenidamente las ideas que Hartog desarrolla sobre la transición entre un régimen de historicidad dominado por la historia magistra hacia el régimen moderno de lo que él denomina presentismo, ${ }^{31} \mathrm{y}$ sus aplicaciones a nuestro objeto de estudio en particular.

En principio, Hartog explica que hasta fines del siglo XVIII, el gran modelo de la historiografía europea fue la historia magistra, en la que la manera de esclarecer el presente se da a través del pasado. ${ }^{32}$ La concepción ciceroniana de la historia como un depositario de ejemplos era funcional a un paso del tiempo marcado por sucesos ya dados en el pasado que se vuelven a repetir. Así,

una relación como ésta con el tiempo y con la historia alienta las aproximaciones, incita a buscar paralelismos entre los Antiguos y los Modernos, y deberá justificar la práctica de la imitación. Ya que la historia es fundamentalmente repetición, la comparación (como investigación e inventario de semejanzas) con la antigüedad es el primer momento, indispensable, de un pronóstico bien construido. ${ }^{33}$

En la historia magistra el ejemplo responde a un ideal pedagógico formativo del cual se desprenden leyes generales de entendimiento de todas las épocas históricas. Por lo tanto, es claro que la legitimación de la imitación se sostiene gracias a la repetibilidad del ejemplo que necesariamente traza un puente entre el pasado y el presente. En los aspectos de la esfera artística aquí tratados, el paralelismo con la historia magistra se

\footnotetext{
${ }^{28}$ Este sentido de clasicismo ha sido desarrollado teóricamente por Richard Shiff, véase nota 18.

${ }^{29}$ Reinhart Koselleck, Futuro, pasado, 15.

${ }^{30}$ François Hartog, Regímenes de historicidad, 15.

${ }^{31} \mathrm{O}$ "el veloz ascenso de la categoría del presente, que ha llevado a imponer la evidencia de un presente omnipotente" (Íbid., 28).

32 Íbid., 61.

33 Íbid., 99.
} 
desprende como evidente. ${ }^{34} \mathrm{El}$ museo de copias en el marco del ámbito formativo es un depósito de ejemplos a seguir, que se reactivan en el presente cada vez que un estudiante los observa y los imita, de modo que, en cierto sentido, se repiten a través de una transposición y una cadena de copias - del original al calco, y del calco al dibujo como ejercicio. Justamente, Hartog afirma que "en la historia magistra, el ejemplo relacionaba el pasado con el futuro a través de la figura del modelo a imitar". ${ }^{35}$ Asimismo, ya hemos comentado el carácter prescriptivo y pedagógico que adoptan estos ejemplos-modelos, "recuerden las leyes que nos controlan" se decía en el extracto que hemos analizado.

No obstante, ¿se puede reducir la dinámica de funcionamiento del museo de reproducciones artísticas en el marco de la esfera artística formativa a una manifestación de la historia magistra vitae? Ciertamente, hay un elemento de innovación necesario latente en el proceso que ya hemos brevemente abordado. El museo de copias muestra el pasado para que el arte progrese hacia el futuro. Y en este sentido, cabe introducir el concepto de progreso, que, según Koselleck en su ensayo titulado "«Progress» and «Decline». An appendix to the history of two concepts" (2002), es una categoría moderna que se hace disponible hacia el siglo XVIII, coincidiendo con el declive de la historia magistra. Si bien Koselleck al definir el concepto de progreso como moderno justamente lo separa de esferas particulares de actuación dado que afirma que el objeto del progreso se universalizó y se convirtió en un colectivo singular, a los fines del presente trabajo cabe redirigir esta definición hacia la esfera artística. Porque en verdad, se podría inclusive afirmar que se dio un proceso generalizado de parangonar el progreso de las artes con el progreso de la civilización entera, conformando una unidad no siempre distinguible: "Así, a partir de las historias individuales de casos de progreso se llegó al progreso de la historia. Esta es una segunda fase. Porque en el curso de la universalización de nuestro concepto, sujeto y objeto intercambian sus roles". ${ }^{36}$ Como afirma Michaud, las artes visuales contenían "el espíritu protector de la nación" y eran "la encarnación visible del espíritu de un pueblo.."; ${ }^{37}$ por lo tanto, progreso en clave moderna y arte se funden en una unidad.

El progreso adquiere en nuestra argumentación una doble pertinencia. Por un lado, en clave futuro, en cuanto a esos artistas que contribuirían al progreso de las artes en el marco del desarrollo de su escuela nacional. Este proceso estuvo enmarcado por un alto grado de asociación entre arte y civilización e implicaba un accionar directo por parte de la nación, de modo que se volvió una acción política del Estado-nación y tendió a traducirse en el fomento de políticas culturales y de apoyo estatal en pos de la institucionalización de las artes.

\footnotetext{
${ }^{34}$ Hartog también hace uso del ámbito artístico para su argumentación pero tomando como objeto el patrimonio. Como ya hemos mencionado, recupera a la historia magistra a través del la noción de patrimonalización: "puede así avanzarse hacia una recuperación del modelo de la historia magistra. Con mayor precisión, a través del intermediario del patrimonio nacional-universal, se pone en movimiento una forma renovada de la historia magistra, en donde buscan articularse el llamado pasado y la apertura hacia el porvenir. Una forma de volver a cerrar la brecha del tiempo o de desenredarla" (Íbid., 208).

35 Íbid., 131. Luego en cambio, el régimen moderno inaugura la noción de acontecimiento único e irrepetible, y el pasado ya no ilumina el futura. Sobre esta temática Koselleck también dice: "La unicidad de los acontecimientos - premisa teórica tanto del historicismo como del progreso- no sabe de la repetibilidad, por lo que no permite ninguna indicación práctica inmediata. En esto, la 'historia' moderna ha destronado a la antigua historia como magistra vitae" (Reinhart Koselleck, Futuro, pasado, 152).

${ }^{36}$ Reinhart Koselleck, The practice of conceptual history, 230.

${ }^{37}$ Éric Michaud, Las invasiones bárbaras, 162.
} 
Pero, por otro lado, en clave pasado, el museo de copias, al tener la función de conformar una historia del arte, también mostraba el progreso al interior de este relato. En la reunión sintética de lo canónico, en un mismo espacio, convivían las manifestaciones del pasado y se escenificaba su devenir. Como ya hemos afirmado, había una conjunción entre las obras maestras y su marco de pertenencia, la escuela artística o estilo. De esta forma, nos encontramos ante un constante vaivén que se da en forma simultánea: entre un pasado único, universal y eterno del canon y de lo clásico y una variación progresiva de innovaciones. ${ }^{38}$ Así, cada pieza inserta en el relato canónico era una unicidad que conformaba un relato que poseía una estructura de repetición. El cambio se integraba a la permanencia porque

[el] progreso, que sólo puede ser pensado como un proceso de tiempo linear, oculta un amplio cimiento de todas aquellas estructuras que han sobrevivido y que, en términos temporales, están basadas en la repetición. Por supuesto que eventos y estructuras están entrelazadas en la realidad histórica. Es la tarea del historiador la de separarlas metodológicamente en la asunción de que no puede discutir ambas al mismo tiempo. ${ }^{39}$

En síntesis, podemos plantear como hipótesis que en el museo de copias se da una construcción de una historia que deriva de una combinación de la historia magistra vitae con el concepto moderno de progreso. Es decir, el pasado se yergue como una lección a seguir, hay un modelo ejemplar prescriptivo que se reproduce a sí mismo a través de la práctica de la copia y la emulación. Este pasado es intrínsecamente funcional a un futuro progreso, es decir, el progreso de las artes -y por ende de la civilización- depende de la correcta aprehensión y elaboración de este pasado. En esta hibridez de aspectos temporales se construye una suerte de modelo para la puesta en práctica de ciertos conceptos de la historia del arte que alcanza a la modernidad.

\section{Consideraciones finales}

Hemos establecido que la dinámica interna de la historia del arte plasmada en el museo de escultura comparada combinó la noción de historia magistra vitae con la de progreso, y fue sumamente funcional a la instancia de formación artística. No obstante, esta estructura convivió con el curso de la producción artística contemporánea y la aparición de estilos, escuelas y grupos que adoptaron múltiples posicionamientos respecto a este canon artístico construido. Se puede plantear que a través del siglo XIX hubo una coincidencia de objetivos y metodologías entre la formación y la producción artística, cuyas divergencias sólo se manifestaron en forma de tensiones múltiples. Luego, con la aparición de las vanguardias artísticas de principios de siglo XX y especialmente con el advenimiento y consolidación del modernismo como relato historiográfico dominante de la historia del arte, este modelo de formación artística comenzó a ser cuestionado desde múltiple frentes. En este sentido, cabe hacer una consideración final sobre la operatividad de la teoría de los estratos del tiempo de Koselleck para pensar la permanencia de ciertas prácticas relativas a la perpetuación del canon y su convivencia con un suceder histórico construido historiográficamente como una sucesión de innovaciones y aparición de novedades. Ciertamente "desde un único curso del tiempo se produce una dinámica de

\footnotetext{
${ }^{38}$ Y también de declives, dado que el relato dominante de la historia del arte se fundamentó en una historiografía basada en el modelo historiográfico de Giorgio Vasari, atravesada por la idea de períodos de declive del arte seguidos por redescubrimientos y renacimiento. Véase Hans Belting, The invisible masterpiece y The end of the history of art? (Chicago: The University of Chicago Press, 1987).

${ }^{39}$ Reinhart Koselleck, The practice of conceptual history, 123.
} 
diversos estratos temporales para el mismo tiempo", ${ }^{40}$ y al momento de analizar estos procesos no se pueden dejar de cotejar estas múltiples instancias discursivas y prácticas que establecen diversos ritmos de acción en relación con la permanencia y el cambio. Sobre esto Koselleck escribe que:

\begin{abstract}
Los acontecimientos difieren unos de otros, pero las condiciones y estructuras de tales sucesos se repiten de forma más o menos continuada. Si consideramos los factores temporales de tales acontecimientos y estructuras, resulta que nos encontramos con aceleraciones y retrasos que producen diferentes velocidades del cambio. Si examinamos a continuación esas diferentes velocidades del cambio, que por supuesto se influyen mutuamente y pueden llegar a producir trastornos catastróficos, nos encontramos, por así decir, con fricciones, fallas o rupturas entre los estratos del tiempo correspondientes a los acontecimientos únicos y aquellos otros estratos que se refieren a las estructuras repetitivas. ${ }^{41}$
\end{abstract}

Por esos motivos, podemos en parte justificar una paradoja sobre el devenir de las colecciones de los museos de calcos: por una parte han permanecido, es hasta el día de hoy que las copias siguen en los pasillos de las escuelas artísticas y ocasionalmente son copiadas o intervenidas. Pero, por otro lado, hubo una ruptura en la concepción de la historia y el tiempo dentro del ámbito artístico (un cambio en el régimen de historicidad diría Hartog) que ha hecho caduca la dinámica de funcionamiento de estas copias, y por lo tanto, se han transfigurado en otra cosa. Inclusive, estos momentos de quiebre en relación con los museos de copias tuvo momentos y episodios álgidos en los cuales muchos de los calcos de colecciones a lo largo del mundo fueron destruidas y sacadas de exhibición, para luego, recientemente volver a ser descubiertas de modo paulatino y en clave patrimonial -bajo la lógica que Hartog denominó de patrimonialización. ${ }^{42}$ Por ende, a lo largo del siglo XX, podemos detectar un cambio en la estructura dado que

\begin{abstract}
no solamente los acontecimientos repentinos y únicos llevan a cabo modificaciones; también las estructuras de larga duración -que parecen estáticas pero que también cambianposibilitan las modificaciones. La ganancia de una teoría de los estratos del tiempo consiste por tanto en poder medir distintas velocidades, aceleraciones o demoras, y hacer así visibles distintos modos de cambio que ponen de manifiesto una gran complejidad temporal. ${ }^{43}$
\end{abstract}

A lo largo de este trabajo hemos privilegiado el análisis de la estructura en mayor medida que la de los eventos y la sucesión cronológica de los múltiples casos de museos de copias. Entender cómo estos espacios construyeron una historia del pasado del arte en función de su instrumentalización para la formación y la producción artística nos ha permitido examinar la puesta en práctica del concepto de canon y de lo clásico. Si bien hemos empleado un alto grado de generalización teórica para realizar esta instancia, la posibilidad de articularla con casos históricos en particular permitirá continuar las argumentaciones aquí presentadas y establecer convergencias y divergencias en relación con las estructuras. Por el momento, cabe concluir con las palabras de Koselleck:

Entonces podemos decir que la historia tan sólo puede ser investigada si las múltiples dimensiones temporales son mantenidas por separado. Quiero repetir mi proposición: los

\footnotetext{
${ }^{40}$ Reinhart Koselleck, Futuro, pasado, 350.

${ }^{41}$ Reinhart Koselleck, "Historia de los conceptos...", 29.

42 Dice: "Pero lo que hace falta es la categoría de monumento histórico que presupone una toma de distancia. Llega un momento en que un monumento puede ser mirado como algo distinto a lo que era o fue durante mucho tiempo: vuelve a ser visible de otra manera, un semióforo portador precisamente de «valores artísticos e histórico»" (François Hartog, Regímenes de historicidad, 187).

${ }^{43}$ Reinhart Koselleck, Los estratos del tiempo: estudios sobre la historia (Barcelona: Paidós, 2001), 38.
} 
eventos y las estructuras están entrelazadas unas con las otras, pero nunca una puede reducirse a la otra. ${ }^{44}$

\section{Bibliografía}

Avery, Henry O., "The Paris School of Fine Arts", Scribner's Magazine, 4 (II) (1887): 387-403.

Belting, Hans, The end of the history of art? (Chicago: The University of Chicago Press, 1987).

Belting, Hans, The invisible masterpiece (London: Reaktion books, 2001).

Beltrán Fortes, José; Méndez Rodríguez, Luis, y Luzón Nogué, José María, Yesos: Gipsoteca de la Universidad de Sevilla (Sevilla: Centro de Iniciativas Culturales de la Universidad de Sevilla, 2015).

Blanco Rivero, José Javier, "La historia de los conceptos de Reinhart Koselleck: conceptos fundamentales, Sattelzeit, temporalidad e histórica", Politeia, 35, 49 (2012): $1-33$.

Bonnet, Alain, L'enseignement des arts au XIXe siècle. La réforme de l'École des BeauxArts de 1863 et la fin du modèle académique (Rennes: Presses Universitaires de Rennes, 2006).

Frederiksen, Rune y Eckart Marchand, eds., Plaster Casts: Making, Collecting, and Displaying from Classical Antiquity to the Present (Berlin: Walter de Gruyter, 2010).

Gallipoli, Milena, Las rutas del yeso. Circulación y consumos globales de calcos escultóricos hacia fines del siglo XIX (Tesis de maestría, IDAES, UNSAM, Buenos Aires, 2017).

Gazda, Elaine K., ed., The Ancient Art of Emulation. Studies in Artistic Originality and the Tradition from the Present to Classical Antiquity (Michigan: The University of Michigan Press, 2000).

Hartog, François, "El presente y el historiador", Anuario TAREA, 1 (2012): 27-41.

Hartog, François, Regímenes de historicidad. Presentismo y experiencias del tiempo (México: Universidad Iberoamericana, 2007).

Haskell, Francis y Nicholas Penny, Taste and the Antique: The Lure of Classical Sculpture. 1500-1900 (New Haven: Yale University Press, 1981).

Koselleck, Reinhart, "Historia de los conceptos y conceptos de historia”, Ayer 53 (2004): $27-45$.

Koselleck, Reinhart, Begriffsgeschichten (Frankfurt am Main: Suhrkamp, 2006).

Koselleck, Reinhart, Futuro, pasado (Barcelona: Paidós, 1993).

\footnotetext{
${ }^{44}$ Reinhart Koselleck, The practice of conceptual history, 126.
} 
Koselleck, Reinhart, Historia y hermenéutica (Barcelona: Paidós, 1997).

Koselleck, Reinhart, Los estratos del tiempo: estudios sobre la historia (Barcelona: Paidós, 2001).

Koselleck, Reinhart, The practice of conceptual history. Timing history, spacing concepts (Stanford: Stanford University Press, 2002).

Malosetti Costa, Laura, selec., Cuadros de viaje. Artistas argentinos en Europa y Estados Unidos (1880-1910) (Buenos Aires: Fondo de Cultura Económica, 2008).

Michaud, Éric, Las invasiones bárbaras. Una genealogía de la historia del arte (Buenos Aires: Adriana Hidalgo, 2017).

Panofsky, Erwin, Renacimiento y renacimientos en el arte occidental (Madrid: Alianza Editorial, 1997).

Potts, Alex J., "Greek Sculpture and Roman Copies I: Anton Raphael Mengs and the Eighteenth Century", Journal of the Warburg and Courtland Institutes, 43 (1980): 150173.

Schreiter, Charlotte, Gipsabgüsse und antike Skulpturen. Präsentation und Kontext (Berlin: Reimer, 2012).

Schwartz, Emmanuel, La chapelle de l'École des Beaux-Arts de Paris (Paris: École Nationale Supérieure des Beaux-Arts, 2002).

Segré, Monique, L'art comme institution. L'École des Beaux-Arts, 19e et 20e siècles (Cachan: Editions de l'École Normale Supérieure de Cachan, 1993).

Settis, Salvatore, El futuro de lo clásico (Madrid: Abada, 2006).

Shiff, Richard, "Phototropism (Figuring the proper)", Studies in the History of Art, 20 (1989): 161-179.

Shiff, Richard, "The original, the imitation, the copy and the spontaneous classic: Theory and painting in Nineteenth-century France”, Yale French Studies, 66 (1984): 27-54.

Tatarkiewicz, Wladislaw, Historia de la estética I: La estética antigua (Madrid: Akal, 1987).

Tatarkiewicz, Wladislaw, Historia de seis ideas. Arte, belleza, forma, creatividad, mímesis, experiencia estética (Madrid: Tecnos, 2001).

VV.AA. "Le moulage. Pratiques historiques et regards contemporains", In Situ, 28 (2016).

Winckelmann, Johann Joachim, Reflexiones sobre la imitación del arte griego en la pintura y la escultura (Barcelona: Nexos, 1987 [1755]). 


\section{Perfil}

Milena Gallipoli es Magíster en Historia del Arte Argentino y Latinoamericano del IDAES-UNSAM, Licenciada con diploma de honor y Profesora en Artes (Orientación Artes Plásticas) por la Facultad de Filosofía y Letras de la Universidad de Buenos Aires. Actualmente realiza el Doctorado en Historia en IDAES-UNSAM y es becaria interna doctoral en el CONICET. Su tema de Tesis es la circulación y las modalidades de usos y exhibición de calcos escultóricos en diversas colecciones de Sudamérica hacia finales del largo siglo XIX.

\section{Profile}

Milena Gallipoli has a Master in Argentinean and Latin-American Art History at the IDAES-UNSAM. She holds a degree in Art History and a Teacher training degree in Art History from the University of Buenos Aires (UBA) with honorary diploma. Currently she is working for her $\mathrm{PhD}$ in History at the UNSAM and has a doctoral grant from the CONICET. Her topic of dissertation focuses on the circulation and functions of plaster cast collections in South America and the establishment of a commercial network of these objects by end of the long nineteenth century.

Fecha de recepción: 15 de enero de 2019

Fecha de aceptación: 25 de marzo de 2020

Publicación: 1 de julio de 2020

Para citar este artículo: Milena Gallipoli, "La temporalidad del canon y su construcción en la historia del arte a través del museo de copias en el siglo XIX", Historiografías, 19 (enero-junio, 2020), pp. 106-121. 